\title{
Correction to Aging and Cancer Prognosis
}

Arvin Haj-Mirzaian , Khashayar Afshari, and Amir Hossein Abdolghaffari

\section{Correction to:}

Nima Rezaei, Correction to Aging and Cancer Prognosis of Cancers https://doi.org/10.1007/978-3-030-50287-4_24

The spelling of the author name was inadvertently published as Arvin Hajmirzaeian in the Table of Contents, List of contributors and Chapter 24.

This has now been amended throughout the book as Arvin Haj-Mirzaian. 